Artículo

Los textos publicados son responsabilidad exclusiva de sus autores

\title{
Bienestar psicológico, resiliencia y ansiedad en estudiantes universitarios
}

\section{Psychological well-being, resilience, and anxiety in college students}

\author{
Donald W. González-Aguilar \\ Maestría en Docencia Universitaria \\ Universidad San Carlos de Guatemala \\ donald.gonzaleza@gmail.com \\ https://orcid.org/0000-0003-4250-7750
}

\section{Referencia}

González-Aguilar, D. W. (2021). Bienestar psicológico, resiliencia y ansiedad en estudiantes universitarios. Revista Guatemalteca de Educación Superior, 4(1), pp 43-58.

DOI: https://doi.org/10.46954/revistages.v4i1.53

Recibido: $15 / 11 / 2020$

Aceptado: 16/01/2021

\section{Resumen}

Las fortalezas psicológicas ayudan a enfrentar los desafíos emergentes y fomentar la salud mental. El objetivo de esta investigación es determinar la asociación de ansiedad, resiliencia con el bienestar psicológico de estudiantes universitarios. El enfoque del estudio fue cuantitativo, no experimental, de corte transversal y alcance correlacional predictivo. Para el análisis se realizó una regresión logística binomial. Se utilizó la escala de bienestar psicológico de Ryff, escala de 10 ítems de ConnorDavidson. Resilience Scale (CD-RISC) y la escala de trastorno de ansiedad generalizada (GAD-2). Participaron 378 alumnos de la universidad pública de Guatemala. La edad $M=22.2(S D=2.11$ ), siendo 252 (66.70\%) mujeres. La razón de posibilidades (OR) para un indicador bajo de trastorno generalizado de ansiedad fue de 2.907 (IC 95\% 1.718 - 4.922) mientras que un indicador alto de resiliencia en 4.816 (IC 95\% 3.052 - 7.601), los cuales 
afectan al bienestar psicológico, con una varianza explicada de

Palabras clave:

bienestar, salud mental, universidad, adaptación, desesperanza
$26.20 \%$ (Nagelkerke $R^{2}$ ). El modelo fue significativo $(p<.001)$. Los resultados sugieren que se debe de prestar más atención a la ansiedad y la resiliencia para el desarrollo y la mejora del bienestar y salud mental durante la COVID-19.

\section{Keywords:}

well-being, mental health, university, adaptation, hopelessness

\section{Abstract}

The psychological strengths help to deal with the challenges that emerge and encourage the mental health. The aim of this investigation is determining the association of anxiety, resilience with the psychological well-being in students at the university. The approaches of this study was quantitative, nonexperimental, cross-sectional and predictive correlational scope. For the analyses it was used a logistic binomial regression. Using the scale Psychological well-beaing by Ryff, scale of 10 items by Connor-Davidson Resilience Scale (CD-RISC) and the generalized anxiety disorder scale (GAD-2). 378 students from the public university of Guatemala participated. The age $M=22.2$ (SD = 2.11), being 252 (66.70\%) women. The odds ratio (OR) for a low indicator of generalized anxiety disorder was 2.907 (IC 95\% 1.718 - 4.922) while a high indicator of resilience 4.816 (IC 95\% 3.052 - 7.601), which affect the psychological well-being, with an explained variance of de $26.20 \%$ (Nagelkerke $R^{2}$ ). The model was significant $(p<.001)$. The results suggest paid more attention to anxiety and resilience for the development and improvement of well-being and mental health during COVID-19.

\section{Introducción}

En los últimos años se ha prestado atención al enfoque de la psicología positiva en relación con las habilidades humanas. El objetivo final de esta corriente psicológica es identificar los métodos y constructos que incrementan el bienestar humano. Por lo tanto, los factores que causan una mayor adaptación a las necesidades y amenazas de la vida son los constructos básicos de este enfoque de estudio.

El bienestar psicológico es un constructo importante para incrementar el potencial de las personas (Howell, 2009), y esto aplica también en el logro de sus metas académicas (Vaez \& 
Laflamme, 2008). Sin embargo, los estudiantes universitarios en ocasiones pueden llegar a ser vulnerables al estrés y al agotamiento dentro del sistema de educación superior, lo que afecta negativamente el rendimiento académico (Bernhard, 2010; Cushman \& West, 2006; Dyrbye et al., 2005; Law, 2010; Morgan \& De Bruin, 2010). Esto ya que, los alumnos, dedican muchas horas a conferencias, prácticas y estudio independiente; necesitan organizar el tiempo, gestionar relaciones y adaptarse a la vida en el campus; mientras que a menudo también se enfrentan a experiencias que son física, emocional y psicológicamente desafiantes (Cushman \& West, 2006; Dyrbye et al, 2013).

Los estudios de Bernhard (2010), Pritchard, Wilson y Yamnitz (2007), mostraron que los estudiantes experimentaron específicamente un aumento de resultados negativos durante su primer año y reportaron niveles más altos de agotamiento que los estudiantes de pregrado y posgrado. No obstante, otras personas resilientes pueden tener resultados positivos 0 experimentar un ajuste exitoso en el entorno de la educación universitaria.

Según Zautra y colaboradores (2010), la resiliencia se define mejor como una adaptación exitosa a circunstancias adversas. Las características personales determinan los procesos de resiliencia conduciendo a resultados saludables después de las situaciones estresantes. Es por esto por lo que los estados psicológicos positivos como la resiliencia pueden ser más importantes para explicar el desempeño en entornos laborales y académicos que los conceptos psicológicos negativos (Salanova et al., 2010).

Por otra parte, durante el 2020 muchos universitarios han experimentado problemas psicológicos como la ansiedad. Esto debido al rápido aumento del número de decesos por motivos de la COVID-19. Sumado a esto, la escalada significativa de la epidemia y los cambios ambientales severos para las universidades de todo el país están afectando de forma directa o indirecta la salud mental de los estudiantes. Ya desde China (Cao et al, 2020) se reporta el impacto de la COVID-19 en la ansiedad en estudiantes universitarios, donde la mayoría tiene altos indicadores de ansiedad en comparación a otros años (Wang et al, 2020). 
En Guatemala el rápido confinamiento impuesto y el temor por el incremento de contagios llevó a la universidad publica a cerrar sus aulas. Esto fue un cambio rotundo en el estudiante que estaba acostumbrado a trabajar de forma presencial. Esta situación es propicia para el aumento del estrés académico y factores de riesgo como el desempleo y el poco acceso a la internet, principalmente en las sedes departamentales (López, 2021).

De acuerdo con lo anteriormente expuesto, se buscó determinar la asociación entre bienestar psicológico, resiliencia y ansiedad en estudiantes de la única Universidad Publica de Guatemala. La recolección de datos se hizo durante los meses de septiembre a noviembre del 2020, mientras aún había algunas restricciones en el país y las clases desarrollándose de forma virtual.

\section{Materiales y métodos}

El estudio tiene enfoque cuantitativo, no experimental, de corte transversal y alcance correlacional predictivo. Debido a las condiciones de distanciamiento social, se trabajó con un muestreo no probabilístico por voluntarios, donde se le pidió a cada alumno su participación por medios digitales como aulas virtuales, WhatsApp, Facebook e Instagram para responder un cuestionario virtual alojado en la plataforma soscisurvey. Contestado por un total de 378 estudiantes.

Para la recolección de datos se utilizaron cuatro instrumentos. El primero fue Escala de bienestar psicológico de Ryff (1989), particularmente la versión propuesta por Diaz y colaboradores (2006). Este instrumento de auto reporte tiene un total de 39 ítems con opciones de respuesta del 1 al 6 , en el que 1 es totalmente en desacuerdo y 6 totalmente de acuerdo. A mayor puntuación, mayor es la presencia de bienestar psicológico.

El segundo se utilizó para medir la resiliencia en una versión breve de la Connor-Davidson Resilience Scale(CD-RISC), siendo la adaptación española de Notario-Pacheco y colaboradores (2011). Está conformada por 10 ítems (los numerados como 1, 
$4,6,7,8,11,14,16,17,19)$ de la escala original elaborada por Connor \& Davidson (2003). La forma de respuesta es una escala tipo Likert de cinco puntos desde 1 (totalmente en desacuerdo) hasta 5 (totalmente de acuerdo).

El tercero es una escala de primera atención médica que detecta indicadores del trastorno de ansiedad generalizada. Los ítems de la escala corresponden a los criterios para diagnosticar el trastorno de ansiedad generalizada basados en el Manual diagnóstico y estadístico de trastornos mentales de la Asociación Estadounidense de Psiquiatría, Cuarta Edición. Cada elemento se califica en una escala Likert de 4 puntos según la frecuencia con la que el encuestado ha experimentado el síntoma en las últimas dos semanas. Las puntuaciones de los ítems varían de 0 (nada) a 3 (casi todos los días), con puntuaciones totales que van de 0 a 21. Las puntuaciones más altas indican síntomas de ansiedad más graves. Por lo general, se utiliza una puntuación de corte de 3 para indicar síntomas de ansiedad clínicamente significativos (Kroenke et al, 2007).

Por último, se aplicó un cuestionario sociodemográfico que incluye variables como: edad, sexo, empleo, estado civil, ciclo de estudio, centro universitario, unidad académica y jornada.

A partir de los datos recolectados se creó una base de datos en el programa de distribución libre Jamovi en su versión 1.6.9.

El análisis estadístico se realizó inicialmente con la distribución de frecuencias y porcentajes de cada variable. También se buscó la confiabilidad de los instrumentos con la prueba Alfa de Crombach's y se hizo el análisis factorial exploratorio en donde únicamente se presenta en este informe la prueba Kaiser-MayerOlkin (KMO) para cada instrumento.

La asociación fue a través de una regresión logística binomial. Se incluyó el modelo y la varianza ajustada (Nagelkerke $R^{2}$ ) de la dependiente. Además, para la hipótesis de la regresión se comprobó por medio de la prueba Hosmer Lemeshow. 
En cuanto a las consideraciones éticas se les informó a todos los participantes por medio del consentimiento informado los beneficios, riesgos, justicia y confidencialidad de los datos.

\section{Resultados}

La edad promedio de los participantes es de $22.2(S D=2.11$ ). La mayoría de las estudiantes son mujeres (66.7\%). La jornada que más aporta es la nocturna (47.6\%). El resto de las variables están dentro de la Tabla 1.

Para la variable dependiente, que es el bienestar subjetivo la $M$ $=126(S D=19.7)$. Su consistencia internar es excelente $(\alpha=.892)$ y su valor de $K M O=.903$. Para las variables independientes los resultados indican que resiliencia tiene una $M=39.3(S D=6.68)$, con un $\alpha=.840$ y un $K M O=.894$. Mientas que para ansiedad la $M=4.63(S D=1.76)$ un $\alpha=.740$ y el $K M O=.50$.

Los instrumentos Ryff y CDRisc-10 fueron convertidos en dicotómicos tomando el punto de corte de la media. Para el GAD-2 su punto de corte fue 3. Los resultados los puede observar en la Tabla 2.

Se realizó una regresión logística binomial para determinar la asociación de la ansiedad y resiliencia con el bienestar psicológico de estudiantes universitarios. El modelo fue estadísticamente significativo, $\chi 2(2, N=378)=67.975, p<.001$; explicó el 26.20 $\%$ (Nagelkerke $R^{2}$ ) de la varianza en el bienestar psicológico y clasificó correctamente el $\mathbf{6 9 . 8 0} \%$ de los casos. La sensibilidad fue del 72.40 \% y la especificidad fue del 68.80 \%. La razón de posibilidades (OR) para un indicador bajo de trastorno generalizado de ansiedad es de 2.907 (IC 95\% 1.718 - 4.922) mientras que un indicador alto de resiliencia en 4.816 (IC 95\% 3.052 - 7.601). Los demás resultados se pueden observan en la Tabla 3.

A partir de la prueba de Hosmer Lemeshow se rechaza la hipótesis nula $(p=.964)$, indicando que las variables dentro del modelo tienen un ajuste adecuado. 


\section{Discusión}

Los resultados de la regresión logística binominal mostraron que altos indicadores de resiliencia y bajos indicadores de trastorno de ansiedad generalizada han sido predictores significativos para bienestar psicológico durante la pandemia de la COVID-19, la cual ha afectado la forma en que las personas viven las relaciones interpersonales. El encierro se ha caracterizado por una organización diferente de la vida diaria, con un incremento del tiempo en el hogar y una reducción de la distancia a través de dispositivos digitales (Saladino et al, 2020).

Además, es un factor adicional por el que los estudiantes deben estar estresados y ansiosos (Pragholapati, 2020). En varios estudios de ansiedad y bienestar psicológico ya sea en la pandemia mundial actual (Alyami et al, 2020; Lai et al, 2020) o pasadas (Taylor et al, 2008; Van Bortel et al, 2016) los hallazgos son que alrededor del $35 \%$ de estudiantes universitarios reportan ansiedad en algún nivel y el $13 \%$ tuvo niveles severos. Tales resultados son diferentes a los obtenidos en este estudio, ya que el $28 \%$ tiene indicadores de trastornos de ansiedad generalizada, aunque este dato puede indicar que los resultados son consistentes con el estado prepandémico. Sin embargo, hay que considerar lo planteado por Cao y colaboradores (2020) quienes refieren la falta de mediciones longitudinales de los niveles de ansiedad. La falta de este tipo de investigaciones podría haber contribuido a los resultados presentados.

Así también, investigaciones previas como la de Johnson (2011) y respalda la relación entre el bienestar psicológico y su relación con ansiedad y resiliencia. Se puede decir que la resiliencia llega a ser una consecuencia del bienestar o una razón que puede conducir a mayores indicadores de bienestar. Estos, no son factores y capacidades estables, pero son factores dinámicos, por lo que se puede inferir que los indicadores de ansiedad afectan la resiliencia y su vez al bienestar psicológico. Así mismo, se sugiere que componentes del bienestar como el dominio ambiental y el propósito de vida podrían predecir los cambios relacionados a la resiliencia de los sujetos. Consecuentemente afectando el aumento o disminución de la resiliencia. 
Por otro lado, los hallazgos del objetivo de estudio respaldan indirectamente a aquellos estudios que demostraron que la resiliencia y el bienestar psicológico es predictiva del éxito académico. Los autores Haktanir y colaboradores (2018), Sattler \& Gershoff (2019) mostraron que los factores de resiliencia contribuyeron al rendimiento académico. Esto quizás se deba que los estudiantes con altos indicadores de resiliencia y bienestar psicológico pueden adaptarse fácilmente al entorno cambiante (Frydenberg, 2004). Como tal, los estudiantes poseen una alta capacidad para recuperarse de situaciones estresantes que a su vez les permiten tener un mayor bienestar y salud mental. Es decir, tienen una fuerte motivación y capacidad para planificar rutas alternativas ante las dificultades y pueden creer que la situación y las dificultades actuales pueden controlarse y superarse. Tener tales creencias puede, en última instancia, permitir que las personas posean una mayor calidad de vida.

Además, Tugade \& Fredrickson (2004), sugirieron que se ha demostrado que resiliencia y bienestar psicológico ayudan a las personas a pasar experiencias indeseables. Algunos investigadores, como explicación probabilística, sugirieron que la resiliencia mejora la autoestima y la oposición exitosa a las experiencias negativas a través del aumento del bienestar psicológico (Benetti \& Kambouropoulos, 2006; Bonanno, 2004). Sobre la base de esta explicación, la resiliencia mediante la mejora de la autoestima, como mecanismo intermedio, puede conducir a una adaptabilidad positiva. Así mismo, menores indicadores de resiliencia pueden llegar a producir una baja autoestima e ineficacia frente a las experiencias negativas. Por lo tanto, las patologías psicológicas, la angustia, la depresión y la ansiedad se asocian a un mal bienestar psicológico.

Es importante remarcar que los estudiantes pueden llegar a sufrir un trastorno de ansiedad generalizada a causa de la pandemia de la COVID-19 (Lee et al., 2014). Por lo que la identificación de factores de protección centrados en la prevención de problemas de salud mental juega un papel crucial para mejorar el bienestar y la salud mental. Por tanto, factores como la resiliencia adquieren protagonismo para amortiguar el impacto negativo de las perturbaciones en tiempos de crisis. Sin embargo, es 
importante que el estudio longitudinal y la práctica clínica evalúen si promover la resiliencia y buscar bajar indicadores de ansiedad puede mejorar el bienestar subjetivo y la salud mental a lo largo del tiempo.

Dado que una de las medidas más importantes para minimizar la propagación del COVID-19 es adoptar el distanciamiento social centrado en la menor cantidad de contacto físico mediante la interrupción de la transmisión, es fundamental diseñar programas de capacitación de resiliencia de forma virtual, que tengan cantidad mínima de contacto con las personas, para contribuir al bienestar psicológico de quienes se encuentran bajo riesgo de COVID-19.

Se debe agregar también que la tecnología ha hecho la vida relativamente fácil, se puede utilizar convenientemente para ofrecer programas de intervención psicológica en línea que se centren en mejorar la salud mental a bajo costo a gran escala en tiempos de crisis sanitaria.

Por otro lado, si bien es imposible preparar completamente a los estudiantes y profesores para una pandemia similar a COVID-19, la resiliencia y el bienestar psicológico pueden ayudar a reducir la ansiedad asociada. Además, los resultados de este estudio brindan información valiosa sobre el estado psicológico de los estudiantes en un momento crucial, y esto, por supuesto, tiene su propio mérito. Sin embargo, es igualmente importante que las investigaciones futuras se centren y sugieran soluciones para abordar los efectos asociados con las pandemias.

Por último, los datos presentados contribuyen a ir cerrando la brecha de estudios relacionados con la psicología y la educación universitaria en Guatemala. Esto podría orientar a los responsables de la formulación de políticas a desarrollar protocolos de gestión de riesgos como parte de su política para reducir patologías psicológicas en los estudiantes universitarios. Aunque, lo más importante es que, por mucho que estemos convencidos de que COVID-19 es el enemigo actual de la humanidad, debemos ser conscientes del impacto asociado y ser capaces de responder de manera efectiva a todas las consecuencias. 
Se debe tomar en cuenta que el estudio ha tenido varias limitaciones. En primer lugar, se trata de una encuesta de estudio transversal realizada en un único momento. Por tanto, es difícil sacar una conclusión sobre efectos causales entre las variables de estudio. En segundo lugar, se utilizó un enfoque en línea para recopilar datos. Las respuestas de las personas sin acceso a Internet podrían estar subrepresentadas. El uso de un método de muestreo por voluntarios para la recopilación de datos fue otra limitación de este estudio. Por tanto, no podemos generalizar los resultados de este estudio a la población. Se recomienda encarecidamente la selección aleatoria de participantes en estudios futuros. En estos estudios, examinar el efecto de las características de la muestra (por ejemplo, el género) también podría ser útil para desarrollar estrategias de intervención para promover la salud mental en estudiantes universitarios. A pesar de esta limitación, La administración de encuestas en línea era la única forma viable de recopilación de datos en tiempos de brote.

\section{Referencias}

Alyami, M., Henning, M., Krägeloh, C. U., \& Alyami, H. (2020). Psychometric evaluation of the Arabic version of the Fear of COVID-19 Scale. International journal of mental health and addiction, 1-14. https://doi.org/10.1007/ s11469-020-00316-x

Benetti, C., \& Kambouropoulos, N. (2006). Affect-regulated indirect effects of trait anxiety and trait resilience on selfesteem. Personality and individual differences, 41(2), 341-352. https://doi.org/10.1016/j.paid.2006.01.015

Bernhard, J. (2010). Improving engineering students' learning through the use of a variation approach: Examples from a research-based learning environment in mechanics. In ReflekTori 2010 (pp. 46-56). DipoliReports. https://www.diva-portal.org/smash/record. jsf?pid=diva2\%3A397177\&dswid=-9005 
Bonanno, G. A. (2004). Loss, Trauma, and Human Resilience:

Have We Underestimated the Human Capacity to Thrive After Extremely Aversive Events? American Psychologist, 59(1), 20-28. https://doi.org/10.1037/0003-

$066 \times .59 .1 .20$

Cao, W., Fang, Z., Hou, G., Han, M., Xu, X., Dong, J., \& Zheng, J. (2020). The psychological impact of the COVID-19 epidemic on college students in China. Psychiatry research, 287, 112934. https://doi.org/10.1016/j. psychres.2020.112934

Connor, K. M., \& Davidson, J. R. (2003). Development of a new resilience scale: The Connor-Davidson resilience scale (CD-RISC). Depression and anxiety, 18(2), 76-82. https:// doi.org/10.1002/da.10113

Cushman, S., \& West, R. (2006). Precursors to college student burnout: Developing a typology of understanding. Qualitative research reports in communication, 7(1), 23-31. https://doi. org/10.1080/17459430600964638

Díaz, D., Rodríguez-Carvajal, R., Blanco, A., Moreno-Jiménez, B., Gallardo, I., Valle, C., \& Van Dierendonck, D. (2006). Adaptación española de las escalas de bienestar psicológico de Ryff. Psicothema, 18(3), 572-577.

Dyrbye, L. N., Thomas, M. R., \& Shanafelt, T. D. (2005). Medical student distress: Causes, consequences, and proposed solutions. Mayo Clinic Proceedings, 80(12), 1613-1622. https://doi.org/10.4065/80.12.1613

Dyrbye, L. N., Thomas, T. M. R., Stanford, M. F., Power, D. V, Eacker, A., Harper, W., Durning, S., Moutier, C., Szydlo, D. W., Novotny, P. J., Sloan, J. A., \& Shanafelt, T. D. (2013). Academia and Clinic Office Practice. Ann Intern Med, 136, 127-135. https://doi.org/10.7326/0003-4819-149-5200809020-00008 
Frydenberg, E. (2004). Coping competencies: What to teach and when. Theory into practice, 43(1), 14-22. https:// doi.org/10.1207/s15430421tip4301_3

Haktanir, A., Watson, J. C., Ermis-Demirtas, H., Karaman, M. A., Freeman, P. D., Kumaran, A., \& Streeter, A. (2018). Resilience, academic self-concept, and college adjustment among first-year students. Journal of College Student Retention: Research, Theory \& Practice, 1521025118810666. https://doi. org/10.1177/1521025118810666

Howell, A. J. (2009). Flourishing: Achievement-related correlates of students' well-being. The Journal of Positive Psychology, 4(1), 1-13. https://doi. org/10.1080/17439760802043459

Johnson, E. L. (2011). Protective factors and levels of resilience among college students (Doctoral dissertation, The University of Alabama).

Kroenke, K., Spitzer, R. L., Williams, J. B., Monahan, P. O., \& Löwe, B. (2007). Anxiety disorders in primary care: prevalence, impairment, comorbidity, and detection. Annals of internal medicine, 146(5), 317-325. https://doi.org/10.7326/0003-4819-146-5-20070306000004

Lai, J., Ma, S., Wang, Y., Cai, Z., Hu, J., Wei, N., Wu, J., Du, H., Chen, T., Li, R., Tan, H., Kang, L., Yao, L., Huang, M., Wang, H., Wang, G., Liu, Z., \& Hu, S. (2020). Factors Associated With Mental Health Outcomes Among Health Care Workers Exposed to Coronavirus Disease 2019. JAMA network open, 3(3), e203976. https://doi. org/10.1001/jamanetworkopen.2020.3976

Law, D. W. (2010). A measure of burnout for business students. Journal of education for business, 85(4), 195 202. https://doi.org/10.1080/08832320903218133 
Lee, J. S., Ahn, Y. S., Jeong, K. S., Chae, J. H., \& Choi, K. S. (2014). Resilience buffers the impact of traumatic events on the development of PTSD symptoms in firefighters. Journal of affective disorders, 162, 128-133. https://doi.org/10.1016/j.jad.2014.02.031

López, K.R. (2021, 01 feb). El dilema de los sancarlistas en sedes departamentales durante la pandemia: "Comer o pagar internet». Plaza Pública. https://www. plazapublica. com.gt/content/el-dilema-de-los-sancarlistas-en-sedesdepartamentales-durante-la-pandemia-comer-o-pagar

Morgan, B., \& De Bruin, K. (2010). The relationship between the big five personality traits and burnout in South African university students. South African Journal of Psychology, 40(2), 182-191. https://doi. org/10.1177/008124631004000208

Pragholapati, A. (2020, May 11). COVID-19 IMPACT ON STUDENTS. PsyArXiv. https://doi.org/10.17605/OSF.IO/ NUYJ9

Pritchard, M. E., Wilson, G. S., \& Yamnitz, B. (2007). What predicts adjustment among college students? A longitudinal panel study. Journal of American college health, 56(1), 15-22. https://doi.org/10.3200/ JACH.56.1.15-22

Ryff, C. D. (1989). Happiness is everything, or is it? Explorations on the meaning of psychological well-being. Journal of Personality and Social Psychology, 57(6), 10691081. https://doi.org/10.1037/0022-3514.57.6.1069

Saladino, V., Algeri, D., \& Auriemma, V. (2020). The psychological and social impact of Covid-19: new perspectives of well-being. Frontiers in psychology, 11, 2550. https://doi.org/10.3389/fpsyg.2020.577684 
Salanova, M., Llorens, S., Cifre, E., Martínez, I. M., \& Schaufeli, W. B. (2003). Perceived collective efficacy, subjective well-being and task performance among electronic work groups: An experimental study. Small Group Research, 34(1), 43-73. https://doi. org/10.1177/1046496402239577

Salanova, M., Schaufeli, W., Martínez, I., \& Bresó, E. (2010). How obstacles and facilitators predict academic performance: The mediating role of study burnout and engagement. Anxiety, stress \& coping, 23(1), 53-70. https://doi.org/10.1080/10615800802609965

Sattler, K., \& Gershoff, E. (2019). Thresholds of resilience and within-and cross-domain academic achievement among children in poverty. Early Childhood Research Quarterly, 46, 87-96. https://doi.org/10.1016/j. ecresq.2018.04.003

Taylor, M. R., Agho, K. E., Stevens, G. J., \& Raphael, B. (2008). Factors influencing psychological distress during a disease epidemic: data from Australia's first outbreak of equine influenza. BMC public health, 8, 347. https://doi. org/10.1186/1471-2458-8-347

Tugade, M. M., \& Fredrickson, B. L. (2004). Resilient Individuals Use Positive Emotions to Bounce Back From Negative Emotional Experiences. Journal of Personality and Social Psychology, 86(2), 320-333. https://doi. org/10.1037/0022-3514.86.2.320

Vaez, M., \& Laflamme, L. (2008). Experienced stress, psychological symptoms, self-rated health and academic achievement: A longitudinal study of Swedish university students. Social Behavior and Personality: an international journal, 36(2), 183-196. https://doi. org/10.2224/sbp.2008.36.2.183

Van Bortel, T., Basnayake, A., Wurie, F., Jambai, M., Koroma, 
A. S., Muana, A. T., Hann, K., Eaton, J., Martin, S., \& Nellums, L. B. (2016). Psychosocial effects of an Ebola outbreak at individual, community and international levels. Bulletin of the World Health Organization, 94(3), 210-214. https://doi.org/10.2471/BLT.15.158543

Wang, C., Pan, R., Wan, X., Tan, Y., Xu, L., Ho, C. S., \& Ho, R. C. (2020). Immediate Psychological Responses and Associated Factors during the Initial Stage of the 2019 Coronavirus Disease (COVID-19) Epidemic among the General Population in China. International journal of environmental research and public health, 17(5), 1729. https://doi.org/10.3390/ijerph17051729

Zautra, A. J., Hall, J. S., \& Murray, K. E. (2010). Resilience: A new definition of health for people and communities. In J. W. Reich, A. J. Zautra, \& J. S. Hall (Eds.), Handbook of adult resilience (p. 3-29). The Guilford Press. 


\section{Sobre el autor}

\section{Donald W. González-Aguilar}

Es Licenciado en Ciencias Psicológicas, especialidad en Psicología Social por la Escuela de Ciencias Psicológicas y Maestrando en Educación Superior en la Facultad de Humanidades, ambos en la Universidad San Carlos de Guatemala (USAC). Entre la experiencia profesional esta ser profesor investigador en la Unidad Profesional de Investigación en la Escuela de Ciencias Psicológicas de la USAC. Coordinador, investigador y auxiliar II de investigación en la Dirección General de Investigación (DIGIUSAC).

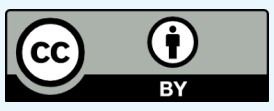

Este texto está protegido por una licencia CreativeCommons 4.0.

Usted es libre para compartir, copiar y redistribuir el material en cualquier medio o formato y adaptar el documento, remezclar, transformar y crear a partir del material para cualquier propósito, incluso comercialmente, siempre que cumpla la condición de atribución: usted debe reconocer el crédito de una obra de manera adecuada, proporcionar un enlace a la licencia, e indicar si se han realizado cambios. Puede hacerlo en cualquier forma razonable, pero no de forma tal que sugiera que tiene el apoyo del licenciante o lo recibe por el uso que hace. 